\title{
A facile, green, one-pot synthesis of amidoalkyl naphthols under solvent-free conditions catalyzed by a carbon-based solid acid
}

\author{
Abolghasem Davoodnia*, Rahil Mahjoobin, Niloofar Tavakoli-Hoseini \\ Department of Chemistry, Faculty of Sciences, Mashhad Branch, Islamic Azad University, Mashhad, Iran
}

\section{A R T I C L E I N F O}

Article history:

Received 18 October 2013

Accepted 25 December 2013

Published 20 April 2014

\section{Keywords:}

Amidoalkyl naphthol

Carbon-based solid acid

Heterogeneous catalysis

Solvent-free condition

\begin{abstract}
A B S T R A C T
An efficient, environmentally friendly procedure for the synthesis of amidoalkyl naphthols through the one-pot, three-component reaction of $\beta$-naphthol, aryl aldehydes, and acetamide in the presence of a carbon-based solid acid under thermal solvent-free conditions is described. The beneficial features of this new synthetic approach include short reaction time, high yields, clean reaction profiles, and a simple work-up procedure. Furthermore, the catalyst can be readily recycled and reused four times without obvious significant loss of activity. The structure of the catalyst was confirmed by Fourier transform infrared spectroscopy, $\mathrm{N}_{2}$ adsorption/desorption analysis, and X-ray diffraction.
\end{abstract}

(c) 2014, Dalian Institute of Chemical Physics, Chinese Academy of Sciences. Published by Elsevier B.V. All rights reserved.

\section{Introduction}

The increasing focus on environmental protection over the last few decades has led both academic and industrial researchers to develop chemical processes which maximize yield and minimum cost while using non-toxic reagents, solvents and catalysts or solvent-free conditions [1,2]. One of the tools used to attain both economic and environmental goals is the multicomponent reaction (MCR) strategy. MCRs have attracted much interest and are highly regarded in modern organic synthesis and medicinal chemistry because they are one-pot processes that bring together three or more components and show high atom economy and high selectivity [3-5]. MCRs have been widely used in the convergent synthesis of complex and important organic molecules from simple and readily available starting materials, and have emerged as powerful tools for drug discovery [6,7]. The development of new MCRs and improvement of known MCRs are therefore areas of considerable current interest. One such reaction is the synthesis of amidoalkyl naphthols. These compounds are generally synthesized via the three-component reaction of an aldehyde, an amide, and $\beta$-naphthol in the presence of various catalysts, such as iodine $[8,9], \mathrm{K}_{5} \mathrm{CoW}_{12} \mathrm{O}_{40} \cdot 3 \mathrm{H}_{2} \mathrm{O}$ [10], sulfamic acid [11], thiamine hydrochloride [12], $\mathrm{Yb}(\mathrm{OTf})_{3}$ in [bmim] $\left[\mathrm{BF}_{4}\right]$ [13], molybdophosphoric acid [14], copper $p$-toluenesulfonate [15], silica supported methanesulfonic acid [16], $\mathrm{Fe}\left(\mathrm{HSO}_{4}\right)_{3}$ [17], $\mathrm{HClO}_{4}-\mathrm{SiO}_{2}$ [18], nano-sulfated zirconia [19], montmorillonite K10 [20], and $\mathrm{Al}\left(\mathrm{H}_{2} \mathrm{PO}_{4}\right)_{3}$ [21]. Although some of these methods have convenient protocols with good to high yields, the majority suffer from at least one of the following disadvantages: unsatisfactory yields, the use of toxic halogenated solvents or catalysts, long reaction time, or the use of expensive catalysts. To avoid these limitations and to improve the reaction conditions available for the synthesis of amidoalkyl naphthols, the exploration of novel methodologies using new heterogeneous and reusable catalysts is still ongoing.

The development of heterogeneous catalysts and the analysis of their effects on specific transformations in chemical syn-

\footnotetext{
* Corresponding author. Tel: +98-511-8435000; Fax: +98-511-8424020; E-mail: adavoodnia@mshdiau.ac.ir, adavoodnia@yahoo.com This work was supported by Islamic Azad University, Mashhad Branch. 
thesis have become a major area of research. The potential advantages of these materials over homogeneous systems, in terms of their simplified recovery and reusability, could potentially allow for the development of environmentally benign chemical procedures in both academic and industrial settings. Catalysts of this type have the potential to make the processes to which they are applied cleaner, safer, higher-yielding, and relatively inexpensive [22-25]. The toxicity and volatile nature of many organic solvents have posed a serious threat to the environment and thus the design of solvent-free catalytic reactions has received significant attention in the area of green synthesis. In addition, reactions performed in the absence of a solvent typically require shorter reaction time and simpler work-up procedures [26,27].

During the course of our recent studies directed towards the development of practical and environmentally friendly procedures for the synthesis of organic compounds using reusable catalysts [28-37], we investigated the application of a carbon-based solid acid (CBSA). This material is easily prepared by heating naphthalene and concentrated sulfuric acid [38] and represents a potential catalyst for a series of organic transformations. This reusable heterogeneous catalyst performed well and showed a high level of catalytic activity in the Mannich [39] and Biginelli [40] reactions, as well as in the synthesis of tetrasubstituted imidazoles [41]. These findings prompted us to investigate the catalytic activity of this material in the synthesis of amidoalkyl naphthols (Scheme 1).

\section{Experimental}

\subsection{Preparation of CBSA catalyst}

Naphthalene (20 g) was heated in concentrated sulfuric acid $\left(>96 \%, 200 \mathrm{ml}\right.$ ) at $250{ }^{\circ} \mathrm{C}$ under a flow of $\mathrm{N}_{2}$. After heating for $15 \mathrm{~h}$, excess sulfuric acid was removed from the dark brown tar by vacuum distillation at $250{ }^{\circ} \mathrm{C}$ for $5 \mathrm{~h}$, resulting in a black solid. The dry solid was subsequently ground to powder and was washed repeatedly in boiling water until impurities such as sulfate ions were no longer detected in the wash water. The density of $\mathrm{SO}_{3} \mathrm{H}$ groups in the product was determined by acid-base potentiometric titration with $\mathrm{NaOH}(0.01 \mathrm{~mol} / \mathrm{L})$ and the concentration of $\mathrm{SO}_{3} \mathrm{H}$ groups attached to the polycyclic aromatic carbon product was found to be $2.81 \mathrm{mmol} / \mathrm{g}$. The resulting black powder was insoluble in common solvents such as water, methanol, ethanol, benzene, and hexane even at their boiling temperatures [38].

\subsection{General procedure for the synthesis of amidoalkyl}

\section{naphthols $\mathbf{4 a - 4 j}$}

A mixture of $\beta$-naphthol 1 ( $2 \mathrm{mmol})$, an aromatic aldehyde 2a-2j (2 mmol), acetamide 3 (2 mmol), and CBSA (0.07 g) was heated in an oil bath at $130{ }^{\circ} \mathrm{C}$ for 2-20 min while monitoring the reaction process by TLC. Upon completion of the transformation, the reaction mixture was cooled to room temperature and hot ethanol was added. This resulted in the precipitation of the catalyst, which was collected by filtration. The product was collected from the filtrate after cooling to room temperature and subsequently recrystallized from ethanol to give compounds $4 \mathbf{4}-\mathbf{4 j}$ (Scheme 1). The melting points of the products were determined using a Stuart SMP3 melting point apparatus. The Fourier transform infrared (FT-IR) spectra of the products were obtained in the form of $\mathrm{KBr}$ disks with a Tensor 27 Bruker spectrophotometer while ${ }^{1} \mathrm{H}$ NMR (500 MHz) spectra were acquired on a Bruker 500 spectrometer, with the following results.

N-[(2-Hydroxynaphthalen-1-yl)(phenyl)methyl]acetamide (4a). ${ }^{1} \mathrm{H}$ NMR (500 MHz, DMSO-d 6 ): $\delta 1.95$ (s, 3H), 7.05-7.25 $(\mathrm{m}, 8 \mathrm{H}), 7.32(\mathrm{t}, 1 \mathrm{H}, J=7.2 \mathrm{~Hz}), 7.73(\mathrm{~d}, 1 \mathrm{H}, J=8.8 \mathrm{~Hz}), 7.77(\mathrm{~d}$, $1 \mathrm{H}, J=7.9 \mathrm{~Hz}$ ), 7.81 (br., 1H), 8.40 (d, 1H, J = 8.3 Hz), 9.95 (s, 1H); FT-IR (KBr, cm-1): $v$ 3408, 3245, 3064, 1639, 1517, 1438, $1370,1338,1275,810,745$.

$\mathrm{N}$-[(4-Bromophenyl)(2-hydroxynaphthalen-1-yl)methyl]ace tamide (4b). ${ }^{1} \mathrm{H}$ NMR (500 MHz, DMSO-d $): \delta 1.97$ (s, 3H), 7.04 (s, 1H), 7.10 (d, 2H, J = 8.3 Hz), 7.19 (d, 1H, J = 8.8 Hz), $7.24(\mathrm{t}$, $1 \mathrm{H}, J=7.4 \mathrm{~Hz}), 7.37(\mathrm{t}, 1 \mathrm{H}, J=7.2 \mathrm{~Hz}), 7.43(\mathrm{~d}, 2 \mathrm{H}, J=8.5 \mathrm{~Hz})$, $7.74(\mathrm{~d}, 1 \mathrm{H}, J=8.8 \mathrm{~Hz}$ ), 7.79 (d, 2H, $J=7.8 \mathrm{~Hz}$ ), 8.55 (br., $1 \mathrm{H}$ ), 10.44 (br., 1H); FT-IR (KBr, cm${ }^{-1}$ ): $v$ 3387, 3064, 2973, 1638, 1516, 1487, 1438, 1370, 1329, 1277, 1072, 1010, 816, 745.

$\mathrm{N}$-[(2-Chlorophenyl)(2-hydroxynaphthalen-1-yl)methyl]ace tamide (4c). ${ }^{1} \mathrm{H}$ NMR (500 MHz, DMSO-d6): $\delta 1.88(\mathrm{~s}, 3 \mathrm{H}$ ), 7.03 (d, $1 \mathrm{H}, J=8.0 \mathrm{~Hz}), 7.08(\mathrm{~d}, 1 \mathrm{H}, J=8.8 \mathrm{~Hz}), 7.17-7.32(\mathrm{~m}, 4 \mathrm{H})$, $7.36(\mathrm{t}, 1 \mathrm{H}, J=7.2 \mathrm{~Hz}), 7.52(\mathrm{~d}, 1 \mathrm{H}, J=7.6 \mathrm{~Hz}), 7.70(\mathrm{~d}, 1 \mathrm{H}, J=$ $8.8 \mathrm{~Hz}), 7.75(\mathrm{~d}, 1 \mathrm{H}, J=7.8 \mathrm{~Hz}), 7.94(\mathrm{~d}, 1 \mathrm{H}, J=8.6 \mathrm{~Hz}), 8.51(\mathrm{~d}$, $1 \mathrm{H}, J=8.0 \mathrm{~Hz}$ ), 9.75 (s, 1H); FT-IR (KBr, cm-1): $v$ 3428, 3062, $1650,1515,1471,1438,1371,1328,1269,809,753$.

$\mathrm{N}$-[(4-Chlorophenyl)(2-hydroxynaphthalen-1-yl)methyl]ace tamide (4d). ${ }^{1} \mathrm{H}$ NMR (500 MHz, DMSO-d 6 ): $\delta 1.99$ (s, 3H), 7.10 (d, $1 \mathrm{H}, J=8.2 \mathrm{~Hz}$ ), $7.16(\mathrm{~d}, 2 \mathrm{H}, J=8.3 \mathrm{~Hz}), 7.22(\mathrm{~d}, 1 \mathrm{H}, J=8.8$ $\mathrm{Hz}$ ), 7.25-7.35 (m, 3H), 7.38 (t, 1H, J= $7.3 \mathrm{~Hz}$ ), 7.78 (d, 1H, $J=$ $8.8 \mathrm{~Hz}$ ), 7.81 (a doublet overlapped with a broad signal, $2 \mathrm{H}, J=$ $7.5 \mathrm{~Hz}), 8.46$ (d, 1H, J = 8.2 Hz), $10.03(\mathrm{~s}, 1 \mathrm{H})$; FT-IR $\left(\mathrm{KBr}, \mathrm{cm}^{-1}\right)$ : $v$ 3392, 3053, 2966, 1638, 1515, 1439, 1374, 1332, 1279, 1244, 1092, 820, 748.

$\mathrm{N}$-[(2,4-Dichlorophenyl)(2-hydroxynaphthalen-1-yl)methyl] acetamide (4e). ${ }^{1} \mathrm{H}$ NMR (500 MHz, DMSO-d6): $\delta 1.89(\mathrm{~s}, 3 \mathrm{H})$,

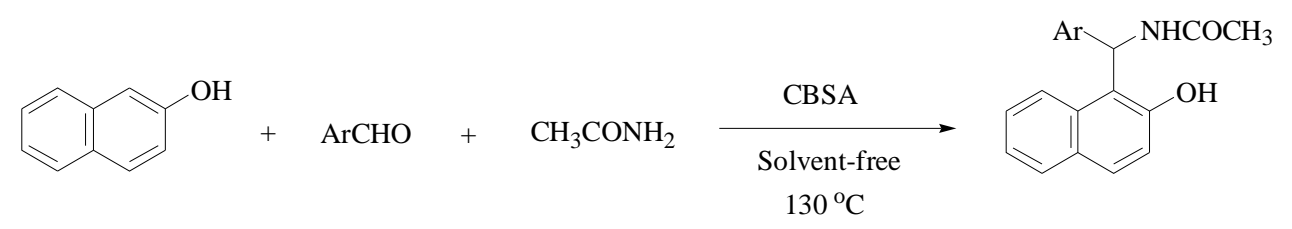


$6.96(\mathrm{~d}, 1 \mathrm{H}, J=7.9 \mathrm{~Hz}), 7.06(\mathrm{~d}, 1 \mathrm{H}, J=8.8 \mathrm{~Hz}), 7.23(\mathrm{t}, 1 \mathrm{H}, J=$ $7.4 \mathrm{~Hz}), 7.35-7.45(\mathrm{~m}, 3 \mathrm{H}), 7.55(\mathrm{~d}, 1 \mathrm{H}, J=8.5 \mathrm{~Hz}), 7.70$ (d, $1 \mathrm{H}, J$ $=8.8 \mathrm{~Hz}), 7.76(\mathrm{~d}, 1 \mathrm{H}, J=7.8 \mathrm{~Hz}), 7.92(\mathrm{~d}, 1 \mathrm{H}, J=8.7 \mathrm{~Hz}), 8.56$ (d, 1H, J = 7.9 Hz), $9.76(\mathrm{~s}, 1 \mathrm{H})$; FT-IR $\left(\mathrm{KBr}, \mathrm{cm}^{-1}\right): v 3405,3118$, $1650,1579,1518,1471,1439,1370,1321,1298,1279,870$, $816,750$.

$\mathrm{N}$-[(4-Fluorophenyl)(2-hydroxynaphthalen-1-yl)methyl]ace tamide (4f). ${ }^{1} \mathrm{H}$ NMR (500 MHz, DMSO-d $): \delta 1.97(\mathrm{~s}, 3 \mathrm{H})$, 7.03-7.10 (m, 3H), 7.13-7.19 (m, 2H), 7.20 (d, 1H, $J=8.8 \mathrm{~Hz}$ ), $7.26(\mathrm{t}, 1 \mathrm{H}, J=7.5 \mathrm{~Hz}), 7.37(\mathrm{t}, 1 \mathrm{H}, J=7.1 \mathrm{~Hz}), 7.76(\mathrm{~d}, 1 \mathrm{H}, J=8.8$ $\mathrm{Hz}$ ), 7.80 (a doublet overlapped with a broad signal, $2 \mathrm{H}, J=7.9$ $\mathrm{Hz}), 8.44(\mathrm{~d}, 1 \mathrm{H}, J=8.2 \mathrm{~Hz}), 10.00(\mathrm{~s}, 1 \mathrm{H})$; FT-IR $\left(\mathrm{KBr}, \mathrm{cm}^{-1}\right): v$ $3393,2975,1628,1509,1439,1377,1335,1279,1226,1159$, $1064,824,749$.

$\mathrm{N}$-[(2-Hydroxynaphthalen-1-yl)(4-methoxyphenyl)methyl]a cetamide (4g). ${ }^{1} \mathrm{H}$ NMR (500 MHz, DMSO-d 6 ): $\delta 1.92(\mathrm{~s}, 3 \mathrm{H})$, $3.64(\mathrm{~s}, 3 \mathrm{H}), 6.77(\mathrm{~d}, 2 \mathrm{H}, J=8.8 \mathrm{~Hz}), 7.00-7.06(\mathrm{~m}, 3 \mathrm{H}), 7.17$ (d, $1 \mathrm{H}, J=8.8 \mathrm{~Hz}), 7.22(\mathrm{t}, 1 \mathrm{H}, J=7.4 \mathrm{~Hz}), 7.32(\mathrm{t}, 1 \mathrm{H}, J=7.6 \mathrm{~Hz})$, 7.71 (d, $1 \mathrm{H}, J=8.8 \mathrm{~Hz}$ ), 7.75 (d, $1 \mathrm{H}, J=7.8 \mathrm{~Hz}$ ), 7.80 (br., $1 \mathrm{H}$ ), $8.36(\mathrm{~d}, 1 \mathrm{H}, J=8.5 \mathrm{~Hz}), 9.91(\mathrm{~s}, 1 \mathrm{H})$; FT-IR $\left(\mathrm{KBr}, \mathrm{cm}^{-1}\right): v 3396$, $3055,2974,1627,1581,1514,1438,1379,1334,1278,1254$, $1177,1043,813,745$.

$\mathrm{N}$-[(2-Hydroxynaphthalen-1-yl)(4-methylphenyl)methyl]ac etamide (4h). ${ }^{1} \mathrm{H}$ NMR (500 MHz, DMSO-d6): $\delta 1.97$ (s, 3H), 2.25 (s, 3H), 7.02-7.07 (m, 4H), 7.09 (d, 1H, J = 8.4 Hz), 7.21 (d, 1H, J $=8.8 \mathrm{~Hz}), 7.25(\mathrm{t}, 1 \mathrm{H}, J=7.4 \mathrm{~Hz}), 7.35(\mathrm{t}, 1 \mathrm{H}, J=7.0 \mathrm{~Hz}), 7.75(\mathrm{~d}$, $1 \mathrm{H}, J=8.8 \mathrm{~Hz}$ ), 7.79 (d, 1H, $J=8.0 \mathrm{~Hz}$ ), 7.83 (br., 1H), 8.39 (d, $1 \mathrm{H}, J=8.4 \mathrm{~Hz}), 9.94(\mathrm{~s}, 1 \mathrm{H})$; FT-IR $\left(\mathrm{KBr}, \mathrm{cm}^{-1}\right): v$ 3397, 3056, $2970,1626,1516,1438,1375,1334,1277,1245,1064,814$, 745.

$\mathrm{N}$-[(2-Hydroxynaphthalen-1-yl)(3-nitrophenyl)methyl]acet amide (4i). ${ }^{1} \mathrm{H}$ NMR (500 MHz, DMSO-d $)$ : $\delta 1.99$ (s, 3H), 7.16 $(\mathrm{d}, 1 \mathrm{H}, J=8.0 \mathrm{~Hz}), 7.19(\mathrm{~d}, 1 \mathrm{H}, J=8.8 \mathrm{~Hz}), 7.25(\mathrm{t}, 1 \mathrm{H}, J=7.6 \mathrm{~Hz})$, $7.38(\mathrm{t}, 1 \mathrm{H}, J=7.4 \mathrm{~Hz}), 7.48-7.58(\mathrm{~m}, 2 \mathrm{H}), 7.75-7.82(\mathrm{~m}, 2 \mathrm{H})$, 7.84 (br., 1H), 7.99 (s, 1H), 8.01 (d, 1H, J = $7.9 \mathrm{~Hz}$ ), 8.58 (d, 1H, $J$ $=8.0 \mathrm{~Hz}), 10.09(\mathrm{~s}, 1 \mathrm{H})$; FT-IR $\left(\mathrm{KBr}, \mathrm{cm}^{-1}\right): v$ 3374, 3198, 3090, $1648,1578,1523,1437,1372,1350,1297,1278,806,747$, $734,713$.

$\mathrm{N}$-[(2-Hydroxynaphthalen-1-yl)(4-nitrophenyl)methyl]acet amide (4j). ${ }^{1} \mathrm{H}$ NMR (500 MHz, DMSO-d 6 ): $\delta 1.98$ (s, 3H), 7.14 $(\mathrm{d}, 1 \mathrm{H}, J=7.9 \mathrm{~Hz}), 7.19(\mathrm{~d}, 1 \mathrm{H}, J=8.8 \mathrm{~Hz}), 7.25(\mathrm{t}, 1 \mathrm{H}, J=7.5 \mathrm{~Hz})$, $7.33-7.40(\mathrm{~m}, 3 \mathrm{H}), 7.74-7.82(\mathrm{~m}, 3 \mathrm{H}), 8.10(\mathrm{~d}, 2 \mathrm{H}, J=8.8 \mathrm{~Hz})$, $8.53(\mathrm{~d}, 1 \mathrm{H}, J=7.9 \mathrm{~Hz}), 10.07(\mathrm{~s}, 1 \mathrm{H})$; FT-IR $\left(\mathrm{KBr}, \mathrm{cm}^{-1}\right): v 3391$, 3050, 2955, 1641, 1523, 1439, 1351, 1281, 1246, 853, 826, 752.

\section{Results and discussion}

\subsection{Characterization results of the catalyst}

The FT-IR spectrum of the catalyst exhibits the $\mathrm{SO}_{2}$ symmetric and asymmetric stretching modes between 1100 and 1250 $\mathrm{cm}^{-1}$ and a broad $\mathrm{OH}$ stretching absorption at $2500-3600 \mathrm{~cm}^{-1}$ (Fig. 1).

Its XRD pattern exhibits a broad and a weak diffraction peaks $\left(2 \theta=10^{\circ}-30^{\circ}, 35^{\circ}-50^{\circ}\right)$ attributable to amorphous carbon (Fig. 2).

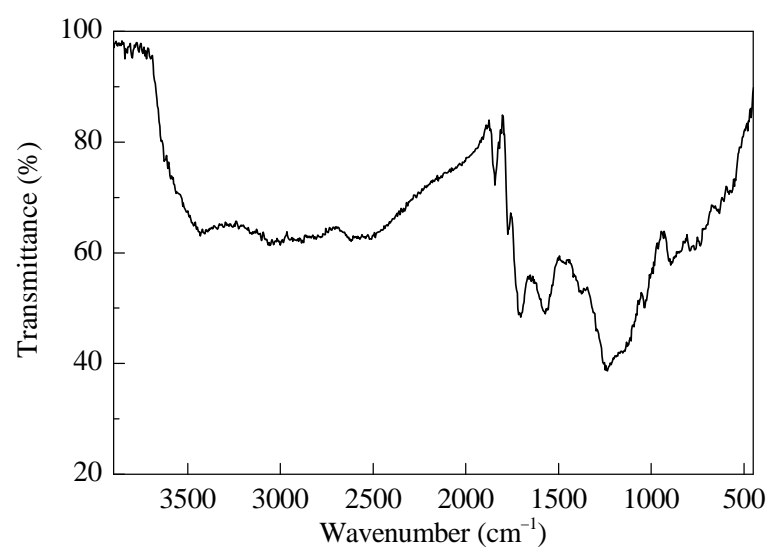

Fig. 1. FT-IR spectrum of the CBSA catalyst.

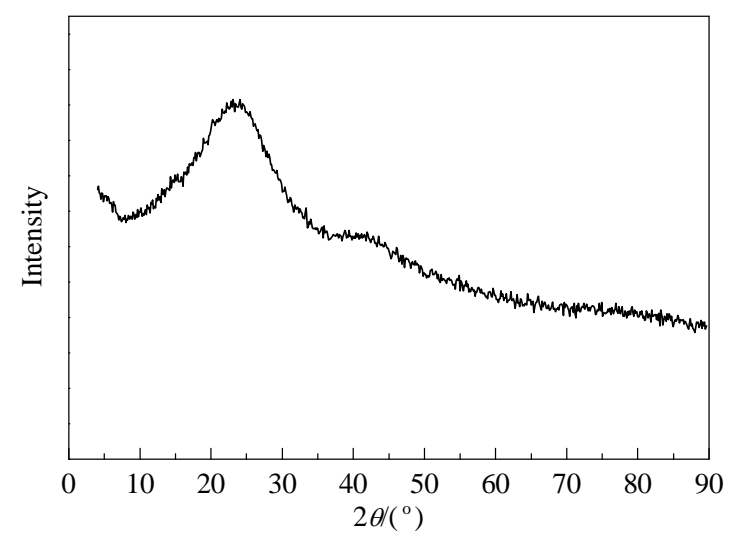

Fig. 2. XRD pattern of the CBSA catalyst.

The $\mathrm{N}_{2}$ adsorption-desorption isotherm of the catalyst is shown in Fig. 3. The isotherm of CBSA catalyst is type II [42], normally obtained with non-porous or macroporous adsorbents. The average pore diameter in the catalyst is $10.1 \mathrm{~nm}$.

\subsection{Synthesis of amidoalkyl naphthols using the CBSA catalyst}

The CBSA catalyst is an amorphous carbon material composed of polycyclic aromatic carbon sheets with attached $\mathrm{SO}_{3} \mathrm{H}$ groups. It functions as a strong solid acid with a high density of acid sites and therefore is able to promote various reactions. Our efforts to develop an efficient and environmentally benign

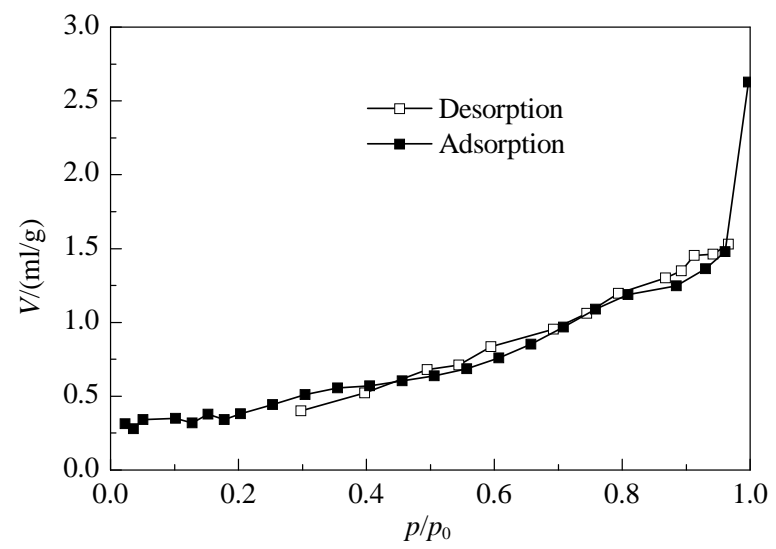

Fig. 3. $\mathrm{N}_{2}$ adsorption-desorption isotherm of the CBSA catalyst. 
methodology for the synthesis of amidoalkyl naphthols initially focused on the three-component condensation of $\beta$-naphthol 1 ( $2 \mathrm{mmol}$ ), 4-chlorobenzaldehyde $\mathbf{2 d}$ ( $2 \mathrm{mmol}$ ), and acetamide $\mathbf{3}$ ( $2 \mathrm{mmol}$ ) as a model reaction. At first, screening trials were performed to optimize various reaction parameters, including catalyst amount, solvent, and temperature, with the results summarized in Table 1 . The reaction was initially carried out without any catalyst at high temperature under solvent-free conditions, following which no product was observed even after prolonged reaction time (Table 1, entry 1). Next, the reaction was performed in the presence of CBSA in different solvents as well as under solvent-free conditions. Among the solvents tested, those being ethanol, methanol, $\mathrm{H}_{2} \mathrm{O}, \mathrm{CH}_{3} \mathrm{CN}$ $\mathrm{CH}_{2} \mathrm{Cl}_{2}, \mathrm{CHCl}_{3}$, and THF, the reaction proceeded most readily to give the highest yield of the product $\mathbf{4 d}$ under solvent-free conditions. The best result was obtained when the reaction was conducted at $130{ }^{\circ} \mathrm{C}$ in the presence of $0.07 \mathrm{~g}$ of the catalyst under solvent-free conditions (Table 1, entry 9). A further increase in temperature and catalyst amount did not improve the product yield.

Encouraged by the remarkable results obtained with the above reaction conditions, and to show the generality and scope of this new protocol, a range of amidoalkyl naphthols were prepared in the presence of CBSA under optimized conditions, with the results shown in Table 2. Most of the reactions proceeded very efficiently and no side-products were observed. As can be seen from Table 2, aromatic aldehydes bearing either electron-donating or electron-withdrawing substituents reacted successfully with $\beta$-naphthol and acetamide to give the corresponding amidoalkyl naphthol products in high yields over short reaction time. The results also show that the aromatic aldehydes with electron-withdrawing groups react more quickly and produce higher yields compared to the aromatic
Table 1

Synthesis of $\mathbf{4 d}$ in the presence of the CBSA catalyst under different reaction conditions.

\begin{tabular}{lccccc}
\hline Entry & $\begin{array}{c}\text { Catalyst } \\
(\mathrm{g})\end{array}$ & Solvent & $T /{ }^{\circ} \mathrm{C}$ & $\begin{array}{r}\text { Time } \\
(\mathrm{min})\end{array}$ & $\begin{array}{c}\text { Isolated } \\
\text { yield (\%) }\end{array}$ \\
\hline 1 & 0.00 & solvent-free & 130 & 120 & None \\
2 & 0.05 & solvent-free & 100 & 15 & 48 \\
3 & 0.05 & solvent-free & 130 & 10 & 84 \\
4 & 0.06 & solvent-free & 100 & 15 & 51 \\
5 & 0.06 & solvent-free & 130 & 10 & 87 \\
6 & 0.07 & solvent-free & 100 & 15 & 57 \\
7 & 0.07 & solvent-free & 110 & 12 & 75 \\
8 & 0.07 & solvent-free & 120 & 10 & 85 \\
9 & 0.07 & solvent-free & 130 & 10 & 90 \\
10 & 0.07 & solvent-free & 140 & 10 & 89 \\
11 & 0.08 & solvent-free & 130 & 10 & 90 \\
12 & 0.09 & solvent-free & 130 & 10 & 90 \\
13 & 0.07 & $\mathrm{C}_{2} \mathrm{H}_{5} \mathrm{OH}$ & reflux & 240 & 33 \\
14 & 0.07 & $\mathrm{CH}_{3} \mathrm{OH}$ & reflux & 240 & 38 \\
15 & 0.07 & $\mathrm{H}_{2} \mathrm{O}$ & reflux & 300 & 15 \\
16 & 0.07 & $\mathrm{CH}_{3} \mathrm{CN}$ & reflux & 270 & 25 \\
17 & 0.07 & $\mathrm{CH}_{2} \mathrm{Cl}{ }_{2}$ & reflux & 300 & 18 \\
18 & 0.07 & $\mathrm{CHCl}_{3}$ & reflux & 330 & 35 \\
19 & 0.07 & $\mathrm{THF}$ & reflux & 240 & 10 \\
\hline
\end{tabular}

Reaction conditions: $\beta$-naphthol 1 ( 2 mmol), 4-chlorobenzaldehyde $\mathbf{2 d}$ ( $2 \mathrm{mmol}$ ), acetamide 3 ( $2 \mathrm{mmol}$ ).

aldehydes with electron-donating groups.

To further evaluate the overall utility of the current methodology, we compared our results with those obtained using other techniques previously reported for the synthesis of amidoalkyl naphthols. As shown in Table 3, it is clear that our method both reduces the required reaction time and generates higher yields of the products.

Table 2

Synthesis of the amidoalkyl naphthols $\mathbf{4 a - 4 j}$ using the CBSA catalyst.

\begin{tabular}{|c|c|c|c|c|c|c|c|c|c|c|c|c|c|}
\hline $\begin{array}{l}\text { En- } \\
\text { try }\end{array}$ & $\mathrm{Ar}$ & Product & & $\begin{array}{l}\text { Time } \\
(\mathrm{min})\end{array}$ & $\begin{array}{c}\text { Isolated } \\
\text { yield (\%) }\end{array}$ & $\begin{array}{c}\text { Melting } \\
\text { point }\left({ }^{\circ} \mathrm{C}\right)\end{array}$ & Entry & $\mathrm{Ar}$ & Product & & $\begin{array}{l}\text { Time } \\
\text { (min) }\end{array}$ & $\begin{array}{c}\text { Isolated } \\
\text { yield (\%) }\end{array}$ & $\begin{array}{c}\text { Melting } \\
\text { point }\left({ }^{\circ} \mathrm{C}\right)\end{array}$ \\
\hline 1 & & & $4 a$ & 5 & 86 & $\begin{array}{c}228-230 \\
(230-232 \\
[14])\end{array}$ & 6 & & & $4 f$ & 10 & 88 & $\begin{array}{c}226-228 \\
(230-232 \\
[17])\end{array}$ \\
\hline 2 & & & $4 b$ & 8 & 90 & $\begin{array}{c}230-232 \\
(227-229 \\
[17])\end{array}$ & 7 & & & $4 g$ & 20 & 86 & $\begin{array}{c}183-185 \\
(180-182 \\
[15])\end{array}$ \\
\hline 3 & & & 4c & 10 & 89 & $\begin{array}{c}212-214 \\
(213-215 \\
[17])\end{array}$ & 8 & & & $4 h$ & 20 & 87 & $\begin{array}{c}215-216 \\
(217-220 \\
[15])\end{array}$ \\
\hline 4 & & & $4 d$ & 10 & 90 & $\begin{array}{c}231-232 \\
(234-237 \\
[15])\end{array}$ & 9 & & & $4 i$ & 3 & 88 & $\begin{array}{c}250-252 \\
(255-257 \\
[15])\end{array}$ \\
\hline 5 & & & $4 e$ & 5 & 86 & $\begin{array}{c}210-212 \\
(201-203 \\
[17])\end{array}$ & 10 & & & $4 j$ & 2 & 93 & $\begin{array}{c}245-247 \\
(42-244 \\
[16])\end{array}$ \\
\hline
\end{tabular}

Reaction conditions: $\beta$-naphthol 1 ( $2 \mathrm{mmol})$, aromatic aldehyde $\mathbf{2 a - 2 j}$ ( $2 \mathrm{mmol})$, acetamide $\mathbf{3}$ ( $2 \mathrm{mmol}), \mathrm{CBSA}(0.07 \mathrm{~g}), 130^{\circ} \mathrm{C}$, solvent-free. 
Table 3

Comparison of the efficiencies of different catalysts for the synthesis of amidoalkyl naphthols.

\begin{tabular}{|c|c|c|c|c|c|}
\hline Catalyst & Solvent & $T /{ }^{\circ} \mathrm{C}$ & Time (min) & Yield (\%) & Ref. \\
\hline $\mathrm{I}_{2}$ & $\mathrm{ClCH}_{2} \mathrm{CH}_{2} \mathrm{Cl}$ & r.t. & $480-1440$ & $62-93$ & [8] \\
\hline $\mathrm{I}_{2}$ & - & 125 & $240-480$ & $70-89$ & [9] \\
\hline $\mathrm{K}_{5} \mathrm{CoW}_{12} \mathrm{O}_{40}-3 \mathrm{H}_{2} \mathrm{O}$ & - & 125 & $120-180$ & $74-90$ & {$[10]$} \\
\hline $\mathrm{K}_{5} \mathrm{CoW}_{12} \mathrm{O}_{40}-3 \mathrm{H}_{2} \mathrm{O}$ & $\mathrm{ClCH}_{2} \mathrm{CH}_{2} \mathrm{Cl}$ & r.t. & $600-720$ & $83-92$ & [10] \\
\hline Sulfamic acid & $\mathrm{ClCH}_{2} \mathrm{CH}_{2} \mathrm{Cl}$ & r.t. & $420-1380$ & $55-86$ & {$[11]$} \\
\hline Thiamine hydrochloride & $\mathrm{C}_{2} \mathrm{H}_{5} \mathrm{OH}$ & 80 & 240 & $75-93$ & [12] \\
\hline $\mathrm{Yb}(\mathrm{OTf})_{3}$ & {$[\mathrm{bmim}]\left[\mathrm{BF}_{4}\right]$} & 80 & $240-480$ & $87-92$ & [13] \\
\hline Molybdophosphoric acid & AcOEt & 65 & $180-240$ & $86-97$ & {$[14]$} \\
\hline Copper $p$-toluenesulfonate & - & 80 & $6-720$ & $16-95$ & [15] \\
\hline Silica supported methanesulfonic acid & - & 80 & $9-420$ & $60-92$ & {$[16]$} \\
\hline $\mathrm{Fe}\left(\mathrm{HSO}_{4}\right)_{3}$ & - & 85 & $25-80$ & $74-97$ & {$[17]$} \\
\hline $\mathrm{HClO}_{4}-\mathrm{SiO}_{2}$ & - & 110 & $30-80$ & $76-95$ & {$[18]$} \\
\hline Nano-sulfated zirconia & - & 120 & $30-90$ & $81-94$ & [19] \\
\hline Montmorillonite K10 & - & 125 & $30-120$ & $65-96$ & {$[20]$} \\
\hline $\mathrm{Al}\left(\mathrm{H}_{2} \mathrm{PO}_{4}\right)_{3}$ & - & 125 & $15-240$ & $55-93$ & [21] \\
\hline CBSA & - & 130 & $2-20$ & $86-93$ & this work \\
\hline
\end{tabular}

The principle advantage of employing heterogeneous solid catalysts in organic transformations is their reusability. The CBSA catalyst was readily recovered from the reaction mixture using the procedure outlined in the experimental section. In this catalyst, $\mathrm{SO}_{3} \mathrm{H}$ groups have been covalently bound to the polycyclic aromatic carbon sheets and, as a result, the catalyst is highly stable and represents a recyclable solid catalyst that does not exhibit the leaching observed with most supported catalysts. The separated catalyst was washed with hot ethanol and then dried at $60{ }^{\circ} \mathrm{C}$ under vacuum for $1 \mathrm{~h}$ before being reused in a similar reaction. We found that the catalyst could be used at least five times with only a slight reduction in activity (Fig. 4).

\section{Conclusions}

The CBSA catalyst is easily prepared from commercially available starting materials, and efficiently catalyzes the synthesis of amidoalkyl naphthols through the condensation of $\beta$-naphthol and aryl aldehydes with acetamide. The advantages of this method include short reaction time, high yields, and easy purification as well as the reusability and economic viability of the catalyst.

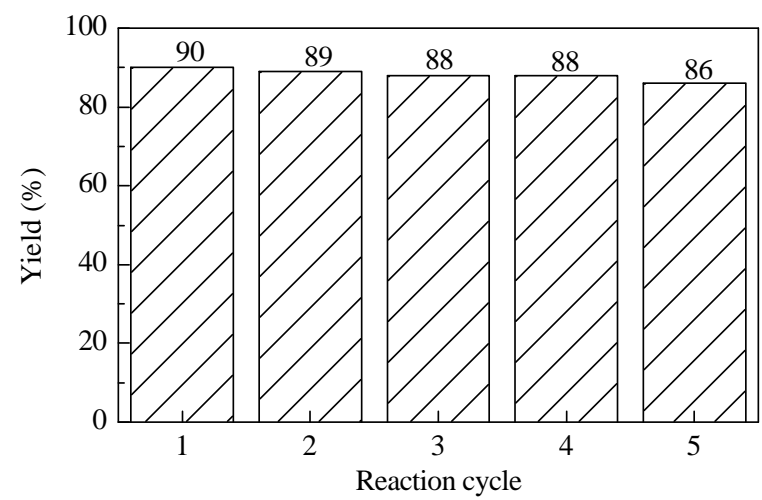

Fig. 4. Reusability of the CBSA catalyst during the synthesis of $\mathbf{4 d}$.

\section{References}

[1] Poliakoff M, Fitzpatrick J M, Farren T R, Anastas P T. Science, 2002, 297: 807

[2] Khojastehnezhad A, Davoodnia A, Bakavoli M, Tavakoli-Hoseini N, Zeinali-Dastmalbaf M. Chin J Chem, 2011, 29: 297

[3] Ugi I. Pure Appl Chem, 2001, 73:187

[4] Dömling A. Chem Rev, 2006, 106: 17

[5] Davoodnia A, Heravi, M M, Safavi-Rad Z, Tavakoli-Hoseini N. Synth Commun, 2010, 40: 2588

[6] Weber L. Drug Discov Today, 2002, 7: 143

[7] Hulme C, Gore V. Curr Med Chem, 2003, 10: 51

[8] Nagawade R R, Shinde D B. Mendeleev Commun, 2007, 17: 299

[9] Das B, Laxminarayana K, Ravikanth B, Rao B R.J Mol Catal A, 2007, 261: 180

[10] Nagarapu L, Baseeruddin M, Apuri S, Kantevari S. Catal Commun, 2007, 8: 1729

[11] Nagawade R R, Shinde D B. Chin J Chem, 2007, 25: 1710

[12] Lei M, Ma L, Hu L H. Tetrahedron Lett, 2009, 50: 6393

[13] Kumar A, Rao M S, Ahmad I, Khungar B. Can J Chem, 2009, 87: 714

[14] Jiang W Q, An L T, Zou J P. Chin J Chem, 2008, 26: 1697

[15] Wang M, Liang Y. Monatsh Chem, 2011, 142: 153

[16] Wang M, Liang Y, Zhang T T, Gao J J. Chin J Chem, 2011, 29: 1656

[17] Shaterian H R, Yarahmadi H, Ghashang M. Bioorg Med Chem Lett, 2008, 18: 788

[18] Shaterian H R, Yarahmadi H, Ghashang M. Tetrahedron, 2008, 64: 1263

[19] Zali A, Shokrolahi A. Chin Chem Lett, 2012, 23: 269

[20] Kantevari S, Vuppalapati S V N, Nagarapu L. Catal Commun, 2007, 8: 1857

[21] Shaterian H R, Amirzadeh A, Khorami F, Ghashang M. Synth Commun, 2008, 38: 2983

[22] Gerard V S, Notheisz F. Heterogeneous Catalysis in Organic Chemistry. San Diego: Elsevier, 2000

[23] Wilson K, Clark J H. Pure Appl Chem, 2000, 72: 1313

[24] Seifi N, Zahedi-Niaki M H, Barzegari M R, Davoodnia A, Zhiani R, Kaju A A. J Mol Catal A, 2006, 260: 77

[25] Zeinali-Dastmalbaf M, Davoodnia A, Heravi M M, Tavakoli-Hoseini N, Khojastehnezhad A, Zamani H A. Bull Korean Chem Soc, 2011, 


\section{Graphical Abstract}

Chin. J. Catal., 2014, 35: 490-495 doi: 10.1016/S1872-2067(14)60011-5

\section{A facile, green, one-pot synthesis of amidoalkyl naphthols under solvent-free conditions catalyzed by a carbon-based solid acid}

Abolghasem Davoodnia*, Rahil Mahjoobin, Niloofar Tavakoli-Hoseini Islamic Azad University, Iran

An efficient method for the synthesis of amidoalkyl naphthols via the solvent-free, one-pot, three-component reaction of $\beta$-naphthol, aryl aldehydes and acetamide in the presence of a carbon-based solid acid (CBSA) as a reusable heterogeneous catalyst is described. The structure of the catalyst was confirmed by FT-IR, BET and XRD techniques.

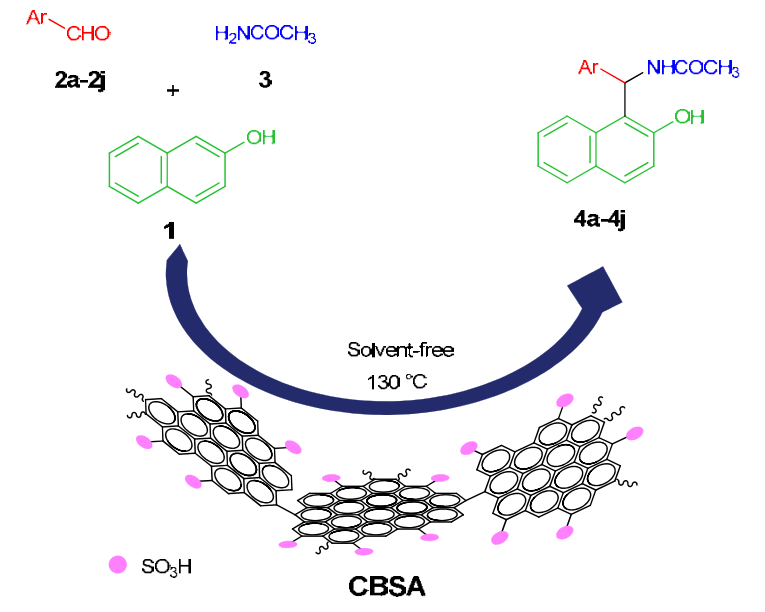

41: 1135

[35] Emrani A, Davoodnia A, Tavakoli-Hoseini N. Bull Korean Chem Soc, 2011, 32: 2385

[36] Davoodnia A, Zare-Bidaki A, Behmadi H. Chin J Catal (催化学报), 2012, 33: 1797

[37] Davoodnia A, Khashi M, Tavakoli-Hoseini N. Chin J Catal (催化学 报), 2013, 34, 1173

[38] Hara M, Yoshida T, Takagaki A, Takata T, Kondo J N, Hayashi S, Domen K. Angew Chem Int Ed, 2004, 43: 2955

[39] Davoodnia A, Tavakoli-Nishaburi A, Tavakoli-Hoseini N. Bull Korean Chem Soc, 2011, 32: 635

[40] Moghaddas M, Davoodnia A, Heravi M M, Tavakoli-Hoseini N. Chin J Catal (催化学报), 2012, 33: 706

[41] Tavakoli-Hoseini N, Davoodnia A. Chin J Chem, 2011, 29: 203

[42] Sing K S W, Everett D H, Haul R A W, Moscou L, Pierotti R A, Rouquerol J, Siemieniewska T. Pure Appl Chem, 1985, 57: 603 